\title{
Hemorrhagic Facet Cyst in the Lumbar Spine Causing Contralateral Leg Symptoms: A Case Report
}

\author{
Risa Utsunomiya, Toshinori Sakai, Keizo Wada, Koichi Sairyo, \\ Hirofumi Kosaka, Shinsuke Katoh, Natsuo Yasui \\ Department of Orthopedics, Institute of Health Biosciences, \\ The University of Tokushima Graduate School, Tokushima, Japan
}

Here we present a case of hemorrhagic lumbar facet cyst presenting with progressive radiculopathy only on the contralat eral side. If a patient has previous back pain or neuropathy for several months and then suddenly deteriorates, hemorrhagic facet cyst of the lumbar spine should be part of the differential diagnosis. However, as in the present case, we should be aware that there is a possibility of a contralateral lesion.

Key Words: Hematoma, Facet cyst, Lumbar spine, Zygapophyseal joint

\section{Introduction}

Symptomatic hemorrhagic facet cyst is a rare condition, and few cases have been reported [1]. Although the exact cause is unclear, it is thought that acute intracystic bleeding could lead to compression of the dural sac and/or nerve roots, and subsequently lead to acute neuropathy [2]. We would expect the lesion to cause neuropathy on the ipsilateral side or on both sides. Indeed, in all previously reported cases neuropathy occurred on the ipsilateral side of the hemorrhagic facet cyst.

In this report, we present a case of hemorrhagic lumbar facet cyst presenting with progressive radiculopathy only on the contralateral side.

\section{Case Report}

The patient was a 65 -year-old woman with hypertension that was well-controlled by medication. There was no history of major trauma or coagulation disorders. She had had no sports- or work-related lumbar trauma. The onset of her mild low back pain was not associated with a traumatic episode. After 2 months, she developed foot drop on the left side. As she was not able to walk, she was referred to our department.

At the initial presentation, muscle weakness of the left leg was observed; the strength of the left tibialis anterior was $2 / 5$, and that of the left extensor hallucis longus was $2 / 5$. No sensory disturbance was found. The left patellar tendon reflex was absent but the right patellar and bilateral ankle jerk reflexes were normal. Bladder and bowel functions were normal. There was no pain or tenderness on her lumbar region. There were no signs of tension of the femoral or sciatic nerves. Coagulation parameters were within the normal range.

Plain radiographs revealed sacralization of the fifth lumbar (L5) vertebra and spondylotic changes (Fig. 1). There was no intervertebral instability. Myelographs showed a block at the L3-L4 level (Fig. 2). Osteoarthritic changes of the L3-L4 facet joints were grade 2 (narrowing plus sclerosis or hypertrophy) according to the classification of Pathria

Received May 12, 2010; 1st Revised Jun 13, 2010 ; Accepted Jun 22, 2010

Corresponding author: Toshinori Sakai, MD, PhD

Department of Orthopedics, Institute of Health Biosciences, The University of Tokushima Graduate School,

3-18-15 Kuramoto-cho, Tokushima 770-8503, Japan

Tel: +81-88-633-7240, Fax:+81-88-633-0178, E-mail: norinoridowluck@yahoo.co.jp 
et al. (Fig. 3) [3].

Sagittal magnetic resonance imaging demonstrated an extradural mass in the right posterolateral spinal canal at the L3-L4 level (Fig. 4). It was an inhomogeneous hyper-intensity mass on T1-weighted images, and a low intensity mass on T2-weighted images. The extradural mass was connected to the right L3-L4 facet joint and compressed the dural sac (Fig. 5).

The patient underwent an L3 laminectomy to decompress the left L4 nerve root and the dural sac, and a right facetectomy at the L3-L4 level to remove the lesion. Additionally, to prevent subsequent instability, posterolateral fusion was done using pedicle screws.

After the paravertebral muscles were retracted, a soft

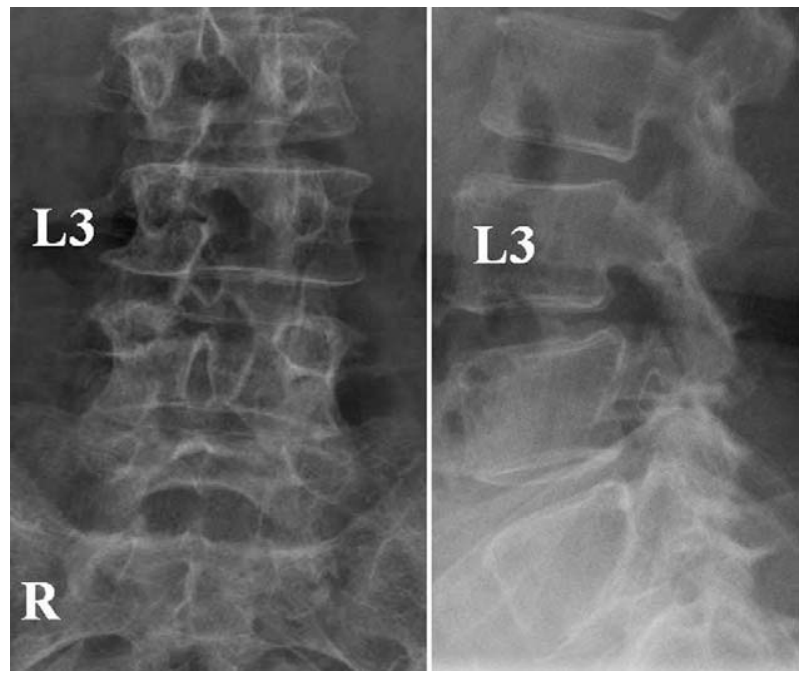

Fig. 1. Plain radiographs showing sacralization of the fifth lumbar (L5) vertebra and spondylotic changes. brownish mass surrounded by a thin capsule was found around the right L4-5 facet joint. Puncture of the mass revealed old coagulated dark-brown blood, but no malignant cells were found on histological examination (Fig. 6). Coagulated blood was found in the extradural space around the right nerve root (Fig. 7). The left nerve root on the symptomatic side was compressed slightly by the hypertrophied ligamentum flavum and facet joint, but there were no inflammatory findings such as adhesion, redness or swelling.

Histologic examination of the surgical specimen showed

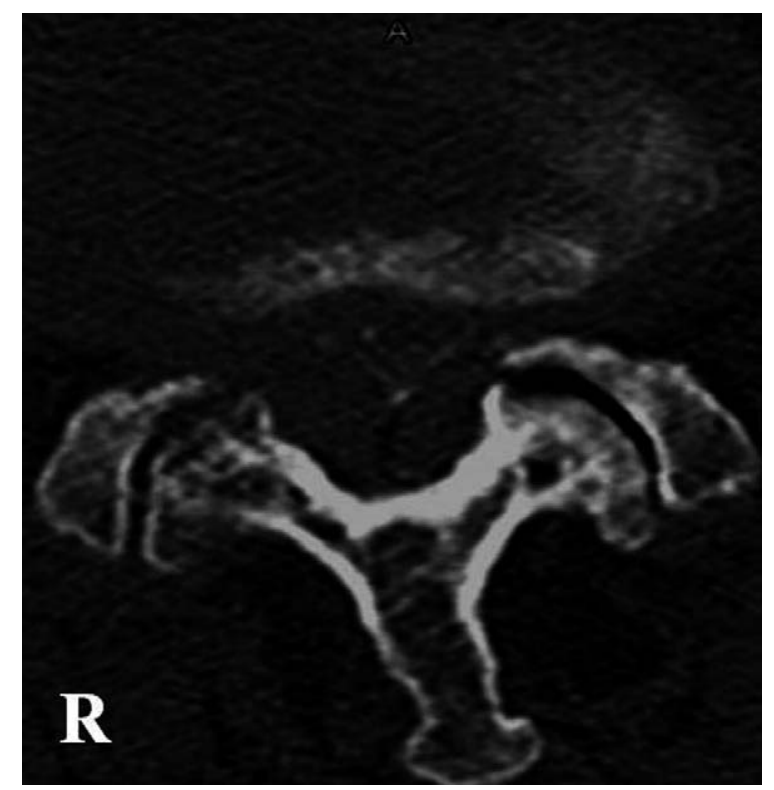

Fig. 3. Computed tomography after a myelogram showing osteoarthritic changes of the L3-L4 facet joints (grade $2=$ narrowing plus sclerosis or hypertrophy) [3].
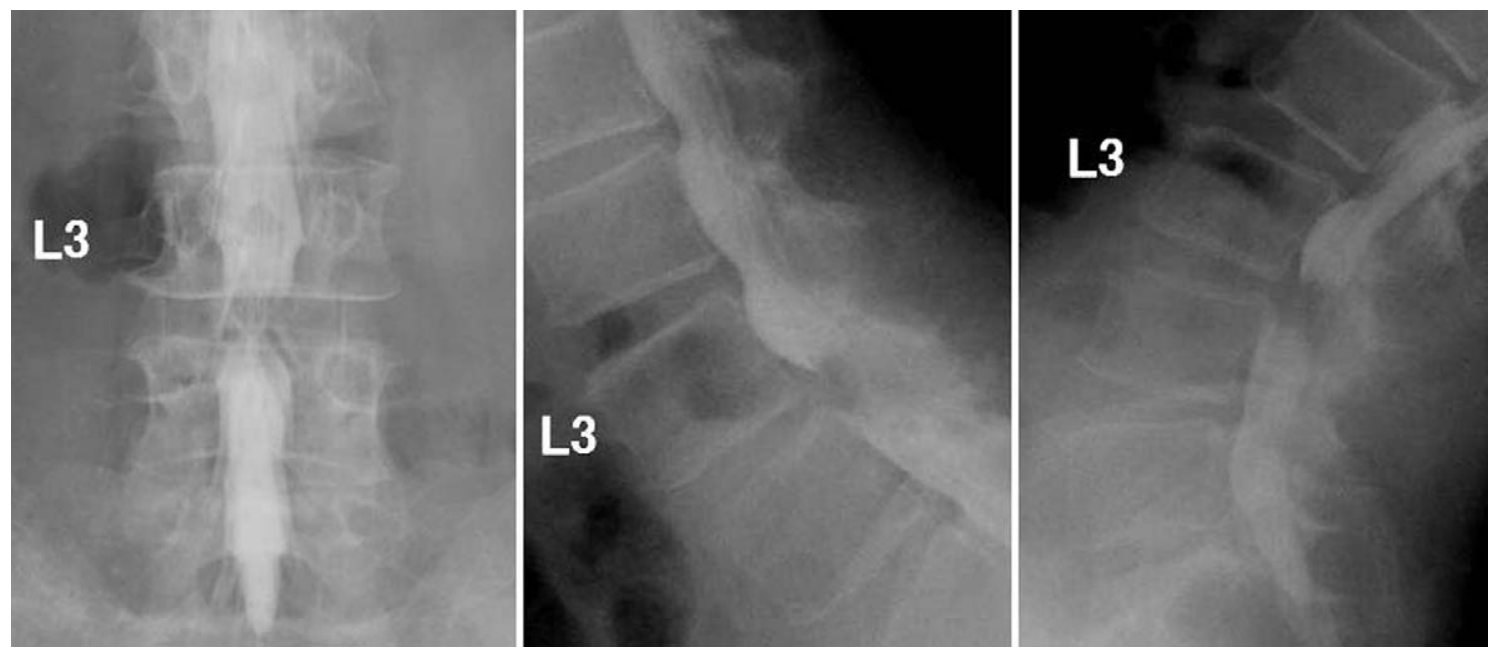

Fig. 2. Myelographs showing a block at the L3-L4 level and no intervertebral instability. 
that the wall formed by fibrous connective tissue contained an old hematoma with hemosiderin deposits (Fig. 8). Typical synovial membranes were absent. Hemosiderin deposits
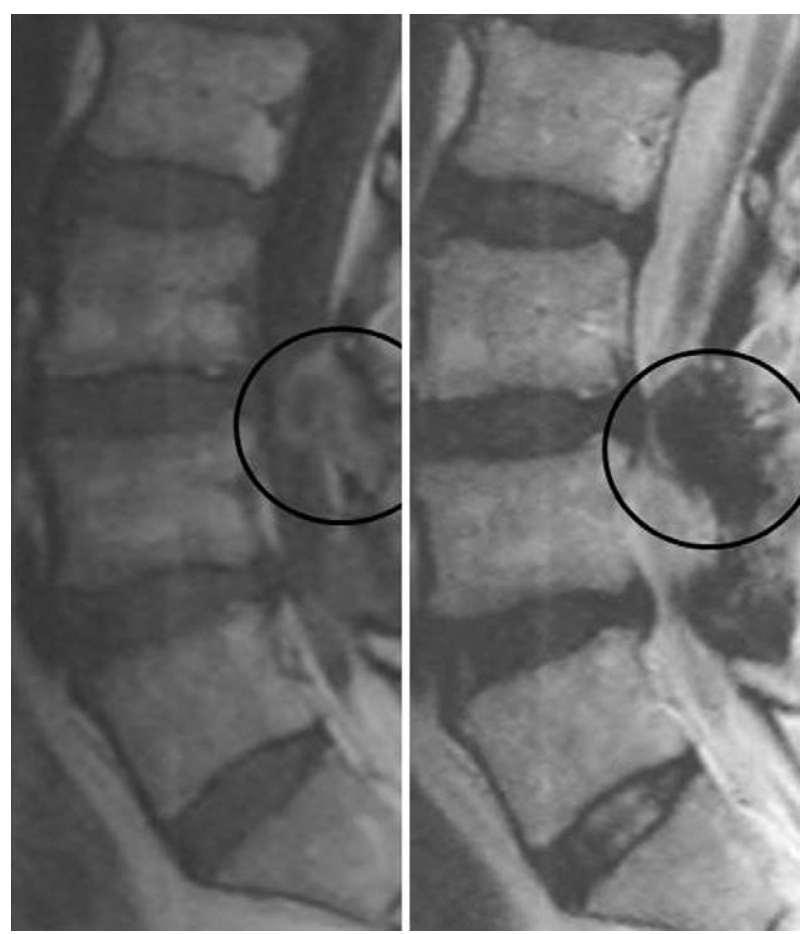

Fig. 4. Sagittal magnetic resonance image demonstrating an extradural mass in the right posterolateral spinal canal at the L3-L4 level. were found in the ventral part of the ligamentum flavum.

After surgery, the patient's symptoms immediately faded. One year later, the left foot drop was completely resolved. Strength of the left tibialis anterior and the left extensor hallucis longus muscles both improved to 5/5. The patient can now walk without aid.

\section{Discussion}

Symptomatic hemorrhagic facet cyst is a rare condition. According to the literature review by Miyatake et al. [1], 32 other cases have been reported. Most patients experienced back pain or neuropathy for several months and then sudden deterioration. The onset of symptoms in the present case was consistent with those of the other reports. Furthermore, in most cases patients were affected at the L3-L4 level or lower, which is consistent with the present case. However, the neuropathy in all reported cases was found on the ipsilateral side of the hemorrhagic facet cyst. The present case is the first report of neuropathy only on the contralateral side to the lesion. According to a few reports regarding contralateral symptoms due to lumbar disc herniation, nerve root anomaly and contralateral compression of the nerve root at the site of the hypertrophied facet joint or pedicle may be the cause of the contralateral symptoms [4-7]. In the present case, although there was no nerve root anomaly, a
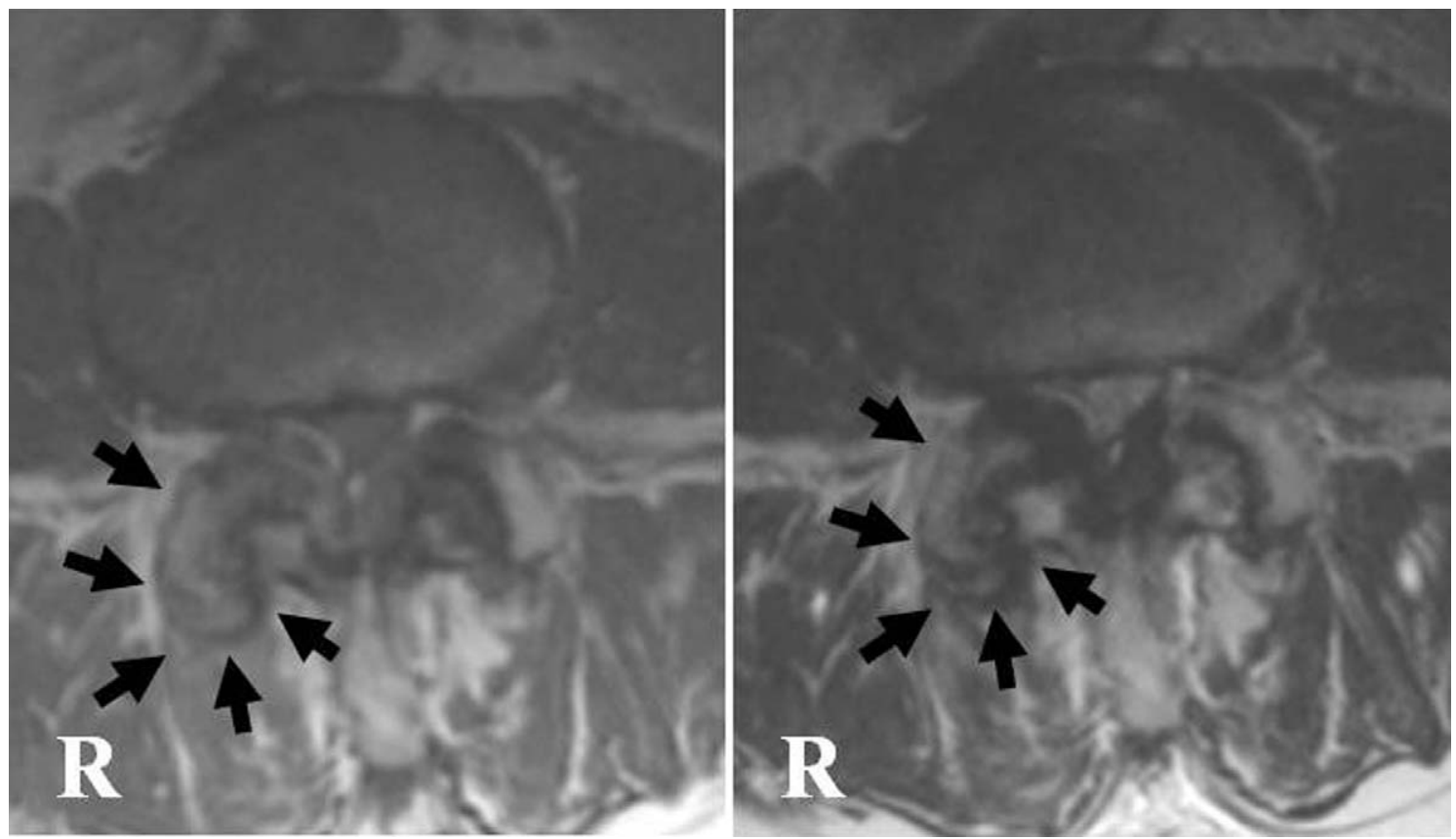

Fig. 5. Mass of inhomogeneous hyper-intensity on T1-weighted images, and of low-intensity on T2-weighted images. The extradural mass lesion was connected to the right L3-L4 facet joint and compressed the dural sac. 

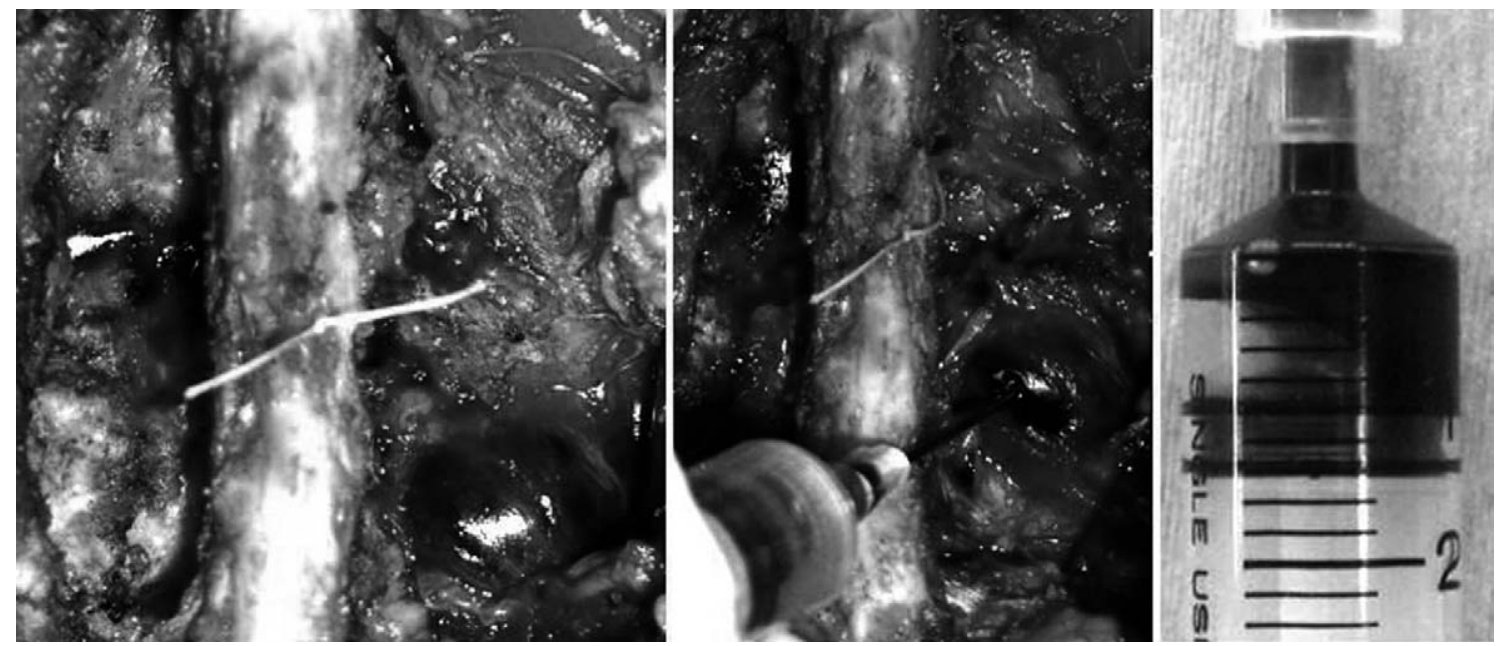

Fig. 6. Intra-operative findings: a solid brownish mass surrounding by a thin facet capsule around the right L4-L5 facet joint. Puncture of the mass revealed old coagulated dark-brown blood, but no malignant cells were found on histological examination.
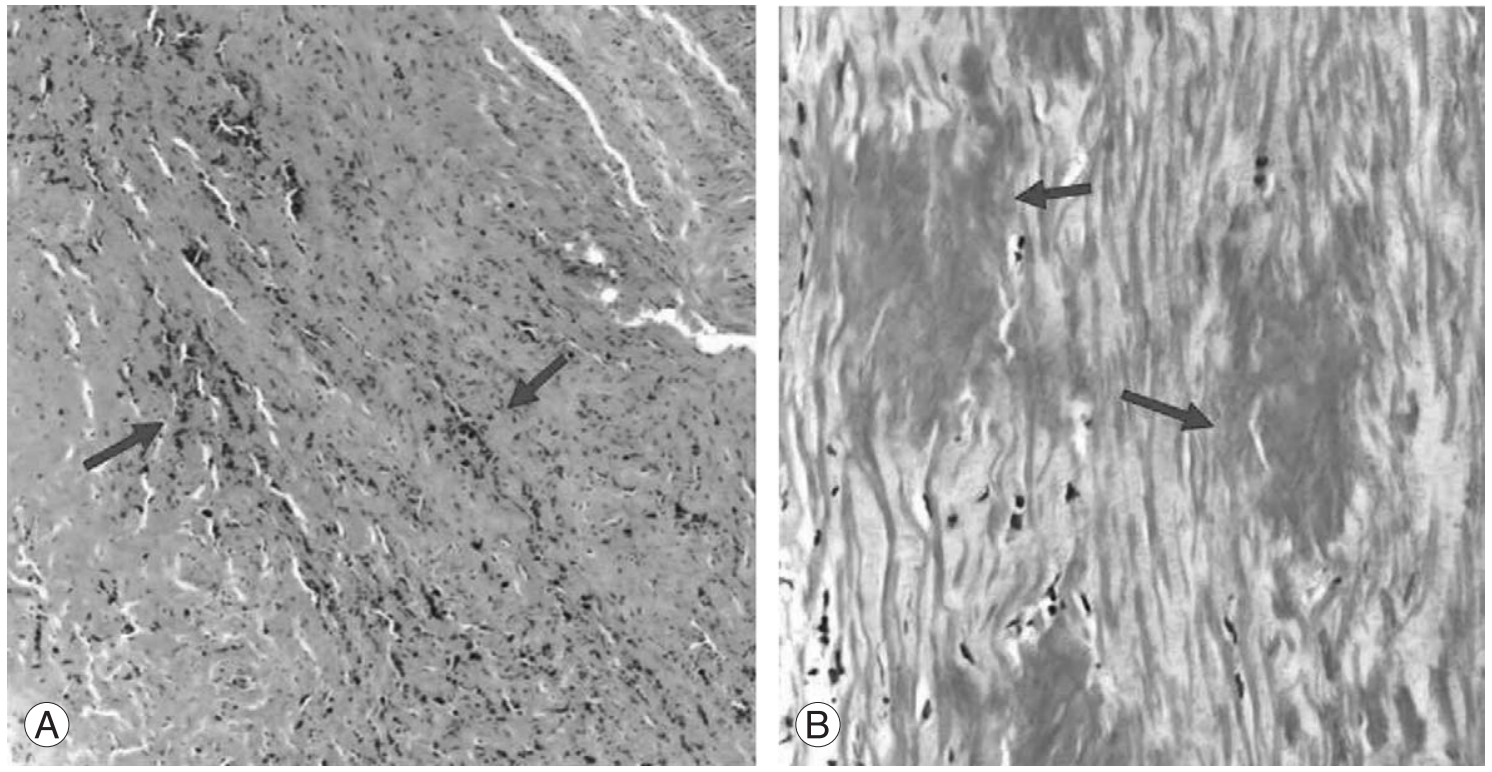

Fig. 8. (A) Histologic examination of the surgical specimen showing that the wall formed by fibrous connective tissue contained an old hematoma with hemosiderin deposits (arrows). Typical synovial membranes were absent. (B) The hemosiderin deposits (arrows) were found in the ventral part of the ligamentum flavum.

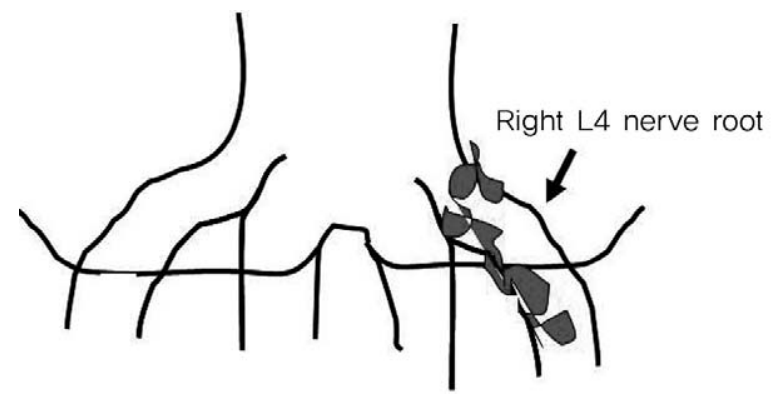

Fig. 7. Coagulated blood was found at the extradural space around the right nerve root. hypertrophied facet joint and ligamentum flavum were found on the symptomatic side. Therefore, rapid progression of canal stenosis caused by the hemorrhagic facet cyst could be one of the factors. Furthermore, in our opinion, blood flow change of the nerve root or ganglion may be another factor [8,9].

This condition is thought to be a primary indication for surgical excision. In about half of the cases reviewed, surgical treatment was deemed urgent because of the severity of symptoms. The presence of fresh intracystic blood and pro- 
gressive palsy indicate the need for rapid surgical treatment. In the present case, we believe that our rapid surgical treatment led to the complete disappearance of the patient's palsy. Thus, patients with a symptomatic hemorrhagic facet cyst should be treated by rapid surgical excision. However, the surgeon should be aware that contralateral hemorrhagic facet cyst of the symptomatic leg, as in the present case, can occur. If this is overlooked, the symptoms could recur.

In the present case, the exact cause of the hemorrhage was not determined. In other reports, the presence of vascular neoendothelium was observed within the cyst formation [2]. However, in the present case, vascular neoendothelium was not observed. Fukuda and Hirofuji [10] suggested three types of peri-articular hemorrhagic change. 1) Hemorrhage into a lumbar facet cyst, including a synovial cyst or ganglion [2,5]. 2) Hematoma of the lumbar facet joint formed directly by bleeding into the facet joint, known as joint apoplexy. 3) Progressive hematoma of the ligamentum flavum. Furthermore, formation of the facet cyst is related to instability of the facet joint [1]. Miyatake et al. [1] speculated that bleeding may come from the synovium in the facet joint or from vessels in the ligamentum flavum. The former is a vascular-rich tissue, the latter has a small arterial network in the interlaminar portion and a venule network in the capsular portion. Once their networks are broken due to instability, blood may enter the pre-existing facet cyst through fissures in the degenerating ligamentum flavum.

In the present case, hemosiderin deposits were histologically found in the ventral part of the ligamentum flavum, and coagulated blood was macroscopically found in the extradural space around the right nerve root. In addition, osteoarthritic changes of the L3-L4 facet joints were grade 2 [3]. Thus, the present case is consistent with the speculation of Miyatake et al. [1].

In conclusion, if a patient has previous back pain or neuropathy for several months with sudden deterioration, hemorrhagic facet cyst of the lumbar spine should be part of the differential diagnosis. However, as in the present case, one should be aware that there is the possibility of a contralateral lesion.

\section{REFERENCES}

1. Miyatake N, Aizawa T, Hyodo H, Sasaki H, Kusakabe T, Sato T. Facet cyst haematoma in the lumbar spine: a report of four cases. J Orthop Surg (Hong Kong) 2009;17:80-4.

2. Tatter SB, Cosgrove GR. Hemorrhage into a lumbar synovial cyst causing an acute cauda equina syndrome: case report. J Neurosurg 1994;81:449-52.

3. Pathria M, Sartoris DJ, Resnick D. Osteoarthritis of the facet joints: accuracy of oblique radiographic assessment. Radiology 1987;164:227-30.

4. Auld AW, DeWall JG. Myelographic defect on the side opposite the leg pain: a case report with an explanation of mechanism of action. Spine (Phila Pa 1976) 1979;4:174-5.

5. Brish A, Payan HM. Lumbar intraspinal extradural ganglion cyst. J Neurol Neurosurg Psychiatry 1972;35:771-5.

6. Choudhury AR, Taylor JC, Worthington BS, Whitaker R. Lumbar radiculopathy contralateral to upper lumbar disc herniation: report of 3 cases. Br J Surg 1978;65:842-4.

7. Nakagawa Y, Yoshida M, Kawakami M, et al. Posterior endoscopic surgery for lumbar disc herniation with contralateral symptoms: a report of two cases. Minim Invasive Neurosurg 2006;49:282-5.

8. Kobayashi S, Takeno K, Miyazaki T, et al. Effects of arterial ischemia and venous congestion on the lumbar nerve root in dogs. J Orthop Res 2008;26:1533-40.

9. Kobayashi S, Mwaka ES, Baba H, et al. Microvascular system of the lumbar dorsal root ganglia in rats. Part II: neurogenic control of intraganglionic blood flow. J Neurosurg Spine 2010;12:203-9.

10. Fukuda C, Hirofuji E. Juxta-facet hematoma. J Orthop Sci 2007;12:597-600. 